\title{
Research Paper \\ The Effect of Eight Weeks of Massage on Blood Pressure, Heart Rate and C- Reactive Protein in Women With Hypertension
}

\author{
*Shahnaz Shahrjerdi ${ }^{1} \mathrm{O}$
}

1. Department of Physiology and Sports Pathology, Faculty of Sport Sciences, Arak University, Arak, Iran.

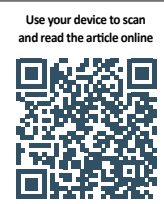

Citation: Shahrjerdi S. [The Effect of Eight Weeks of Massage on Blood Pressure, Heart Rate and C-Reactive Protein in Women With Hypertension (Persian)]. Journal of Arak University of Medical Sciences (JAMS). 2020; 23(3):398-411. https://doi. org/10.32598/JAMS.23.3.620.3

https://doi.org/10.32598/JAMS.23.3.620.3

Key words:

Massage, Systolic and diastolic blood pressure, Heart Rate, C-reactive protein

\section{A B STRACT}

Background and Aim Hypertension is a common disease and universal that can cause cardiovascular disease and kidney damage. The purpose of this study was to determine the effect of an eight-weeks massage on blood pressure (systolic and diastolic), heart rate and C-reactive protein in women with hypertension.

Methods \& Materials In this quasi-experimental study, 44 volunteer women with Mean \pm SD age of $42.12 \pm 5.31$ years were selected from women referred to Arak Oil Company specialized polyclinic with hypertension. Four women excluded from the study for some reason, and the rest were divided into experimental and control groups. In massage group massage was done for three sessions per week, 45-60 minutes in each session, on the back and upper limbs for eight weeks. Data analysis was conducted using dependent and independent $t$-test by SPSS V. 25 statistical software at the significant level of $(P \leq 0.05)$. Ethical Considerations This study (Code: 92-160-26) was approved in Research Ethics Committee of Arak University of Medical Sciences.

Results The results showed that eight weeks of massage reduced blood pressure $(P=0.001)$, the heart rate $(P=0.001)$, and $C$-reactive protein $(P=0.001)$ in women with hypertension compared to the control group $(P=0.62)$. Conclusion The findings of this study showed that massage for eight weeks is an efficient and appropriate method to improve systolic and diastolic blood pressure, heart rate, and C-reactive protein in patients with hypertension.

\section{Extended Abstract}

\section{Introduction}

H

ypertension is one of the most important risk factors for the development of cardiovascular disease, kidney failure which causes great costs for the individual and society, and in general in Iran 25 to $35 \%$ of middle-aged adults with Are hypertensive $[5,7]$.
The mechanism of massage on the physiopathology of hypertension is that following relaxation by massage movements in response to the parasympathetic nerve, it reduces the heartbeat rate and blood pressure [12]. As a hypothesis, inflammation is a factor in increasing blood pressure [13]. High levels of C-reactive protein as an inflammatory indicator can be a risk factor for cardiovascular disease in people with hypertension $[11,13]$, which increases in overweight and obese people [15]. The aim of this study was to investigate the

\section{* Corresponding Author:}

Shahnaz Shahrjerdi, PhD.

Address: Department of Physiology and Sports Pathology, Faculty of Sport Sciences, Arak University, Arak, Iran.

Tel: +98 (863) 4173492

E-mail: s-shahrjerdi@araku.ac.ir 
Table 1. Demographic information of the studied groups

\begin{tabular}{ccc}
\hline & \multicolumn{2}{c}{ Mean \pm SD } \\
\cline { 2 - 3 } Specifications & Massage & Control \\
\hline Age $(\mathrm{y})$ & $43.12 \pm 5.21$ & $45.1 \pm 5.14$ \\
\hline Weight $(\mathrm{kg})$ & $78.5 \pm 5.71$ & $80.8 \pm 5.21$ \\
\hline Height $(\mathrm{cm})$ & $164 \pm 3.03$ & $160.5 \pm 3.22$ \\
\hline Body mass index (kg/m2) & $29.5 \pm 1.81$ & $31.2 \pm 1.72$ \\
\hline waist-to-hip ratio & $87.27 \pm 6.21$ & $90.12 \pm 6.52$ \\
\hline Duration of illness (months) & $44.5 \pm 6.21$ & $40.7 \pm 6.51$ \\
\hline
\end{tabular}

effect of eight weeks of massage on systolic and diastolic blood pressure, heart rate and C-reactive protein in women with hypertension.

\section{Materials and Methods}

This quasi-experimental study was performed on a statistical population with hypertension referred to the specialized polyclinic of Arak Oil Industry. During 3 months of reporting by specialized physicians, 44 female volunteers aged 35-55 years, with a mean Body Mass Index (BMI) of $30.3 \pm 1.54 \mathrm{~kg} / \mathrm{m} 2$, and with mild systolic and diastolic blood pressure of more than 142.4 and $85.6 \mathrm{mmHg}$, were selected, 4 of whom withdrew from cooperation for some reason, and the rest were selected by available sampling method and placed in two groups of "experimental massage" (20 people) and "control" (20 people).

At the beginning, personal information questionnaires were completed and then in the pre-test and post-test stages, blood pressure, heart rate and hs-CRP ${ }^{1}$ test were measured in both groups. Massage protocol was performed during 8 weeks ( 3 sessions per week and each session for 45-60 minutes), including effleurage, petrissage, friction, vibration and tapotement techniques on the whole body. After collecting and entering the data in SPSS software V. 25, the results were analyzed and the Mean \pm SD of the data were calculated using descriptive statistics. A significance level of 95\% $(\mathrm{P} \leq 0.05)$ was considered.

1. high-sensitivity C-reactive protein

Table 2. Blood pressure and heart beat rate values of the massage and control groups in pre-test and post-test stages

\begin{tabular}{|c|c|c|c|c|c|}
\hline \multirow{3}{*}{ Variables } & \multirow{3}{*}{ Groups } & & & \multirow{2}{*}{ Intragroup Changes } & \multirow{2}{*}{ Intergroup Changes } \\
\hline & & \multicolumn{2}{|c|}{ Stages } & & \\
\hline & & Pre-test & Post-test & & \\
\hline Systolic blood pressure & Experimental & $15.3 \pm 150.4$ & $12.4 \pm 125.2$ & $0.001 *$ & $0.001 *$ \\
\hline (mmHg) & Control & $10.5 \pm 145.6$ & $10.3 \pm 140.8$ & 0.62 & - \\
\hline Diastolic blood pressure & Experimental & $0.86 \pm 95.5$ & $0.82 \pm 90.3$ & $0.001 *$ & $0.001^{*}$ \\
\hline (mmHg) & Control & $0.95 \pm 90.7$ & $0.74 \pm 89.2$ & 0.34 & \\
\hline Heart beat & Experimental & $3.24 \pm 105$ & $4.35 \pm 89$ & $0.001^{*}$ & $0.001^{*}$ \\
\hline (beats per min) & Control & $2.26 \pm 95$ & $3.34 \pm 92$ & 0.58 & - \\
\hline
\end{tabular}

* Significance level is considered as $\mathrm{P}<0.05$ 
Table 3. Amounts of C-reactive protein in the massage and control groups during pre-test and post-test

\begin{tabular}{|c|c|c|c|c|c|}
\hline \multirow{3}{*}{ Variables } & \multirow{3}{*}{ Groups } & \multicolumn{2}{|c|}{ Mean士SD } & \multirow{2}{*}{ Intragroup Changes } & \multirow{2}{*}{ Intergroup Changes } \\
\hline & & \multicolumn{2}{|c|}{ Stages } & & \\
\hline & & Pre-test & Post-test & & \\
\hline C-reactive protein & Experimental & $2.24 \pm 5.55$ & 1.442 .91 & $0.001 *$ & $0.001 *$ \\
\hline hs-CRP (mg/L) & Control & 3.96 .1 & 3.516 .5 & 0.62 & - \\
\hline
\end{tabular}

\section{Results}

Demographic information of patients with hypertension in terms of age, weight, height, BMI, Waist-to-Hip Ratio (WHR) and duration of disease is given in Table 1. In terms of demographic information, there was no significant difference between weight, height and BMI.

Table 2 shows the decrease in systolic and diastolic blood pressure before and after the massage intervention with the control group. Also, the massage program had a more significant effect on reducing the serum level of C-reactive protein than the control group $(\mathrm{P} \leq 0.05)$.

Table 3 shows that the massage program had a significant effect on systolic and diastolic blood pressure in women with hypertension $(\mathrm{P} \leq 0.05)$. In other words, the massage program had a more favorable effect on reducing systolic and diastolic blood pressure compared to the control group $(\mathrm{P} \leq 0.05)$. Mean $\pm \mathrm{SD}$ of systolic and diastolic blood pressure in the experimental group before the activity were $150.4 \pm 15.3$ and $95.5 \pm 0.86 \mathrm{mmHg}$, respectively, and after the activity was decreased to $125.2 \pm 12.4$ and $90.3 \pm 0.82 \mathrm{mmHg}$, respectively

The results of the analysis showed that by comparing the mean systolic and diastolic blood pressure of the control and experimental groups, there was a statistically significant difference $(\mathrm{P} \leq 0.05)$. The results of this study were consistent with the findings of previous studies, including Al-Tu'maa et al. [18].

\section{Discussion}

Prolonged hypertension eventually damages arteries throughout the body, including the arteries of vital organs such as the brain, kidneys, heart, and eyes. In this study, systolic and diastolic blood pressure as well as C-reactive protein in the experimental (massage) group were significantly reduced compared to the control group [20]. The present study was consistent with the study of Aourella M (2005), which performed 5 weeks of massage on 30 patients with hypertension, the results of which were a reduction in systolic and diastolic blood pressure as well as stress control [22].

The present study was also consistent with the study of Skuriti et al. (2018), which included 10 minutes of back and leg massage for women and men aged 4060 years admitted to the hospital care ward [23]. But it was not consistent with the study of Bost and Wallis (2006) who performed the effect of 15 minutes of massage once a week on patients with hypertension. Massage reduced stress and anxiety but had no effect on systolic and diastolic blood pressure. The reason for this discrepancy could be related to the number of massage sessions and its duration [25]. Also, the effect of massage on the amount of C-reactive protein was consistent with the research of Sheikhsaraf et al. (2015) who compared aerobic exercise with massage on Creactive protein [29].

Massage stimulates pressure receptors and nerves in internal organs such as the liver and artery walls, reduces sympathetic activity and increases parasympathetic activity, and ultimately reduces inflammation and C-reactive protein [30]. As a result, massage can be used as a non-pharmacological action like other complementary and alternative therapies, in addition to being an economical method and in almost all cases it does not have any serious side effects or drug interactions.

\section{Ethical Considerations}

\section{Compliance with ethical guidelines}

This study was approved by the Ethics Committee of Arak University of Medical Sciences (Code 92-160-26). 
Funding

Arak University of Medical Sciences financially supported this study (Code: 12104/92).

Conflicts of interest

The author declared no conflict of interest.

Acknowledgements

The author is very grateful for the efforts of our colleagues in the Oil Industry Polyclinic of Markazi Province, as well as the patients who encouraged us with their trust and support. 
This Page Intentionally Left Blank 


\title{
تأثير هشت هفته ماساز بر ميزان فشار خون، ضربان قلب و يروتئين واكنشكَر C زنان مبتلا به يرفشارى خون
}

'شهناز شهرجردى'

1. كروه فيزيولوزى و آسيب شناسى ورزشى، دانشكده علوم ورزشى، دانشعاه اراك، اراك، ايران.

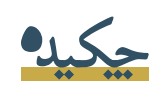

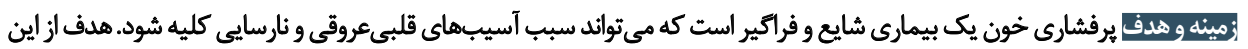

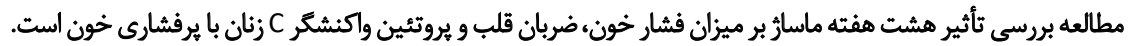

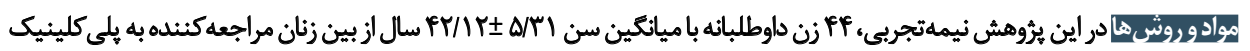

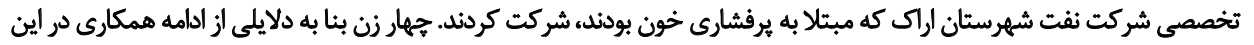

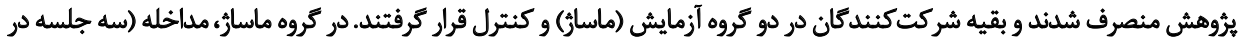

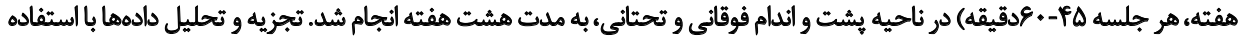

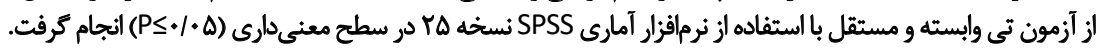

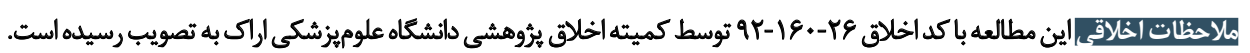

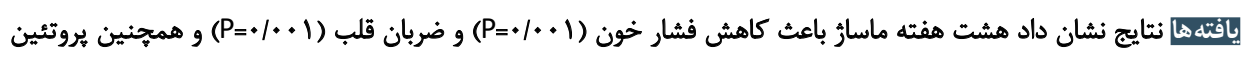

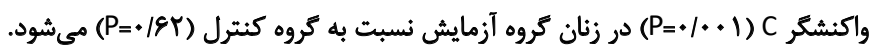

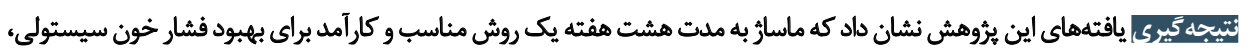

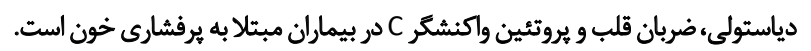

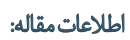

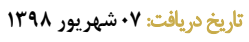

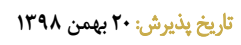

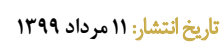

$$
\text { كاسيليدواثرها: }
$$

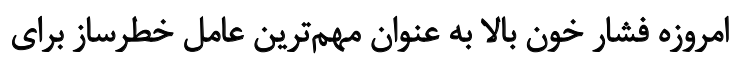

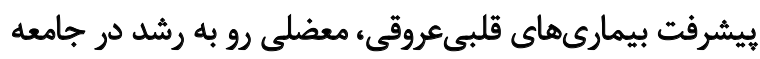

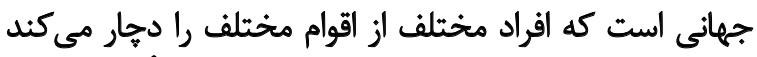

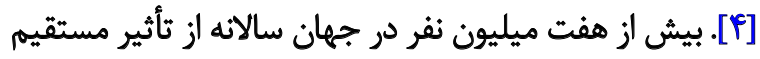

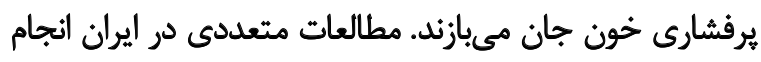

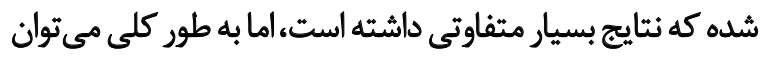

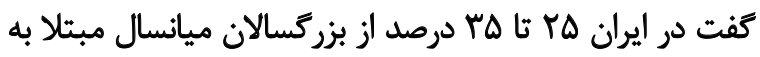

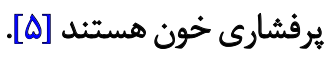

مقدمه

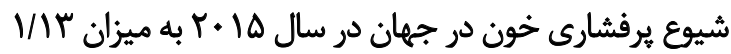

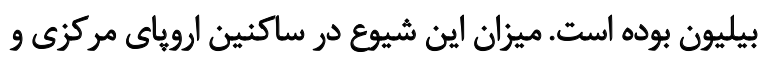

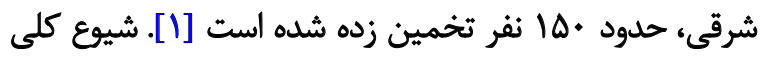

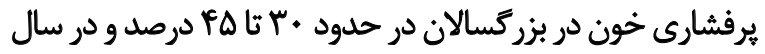

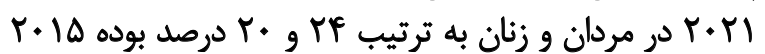

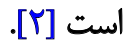

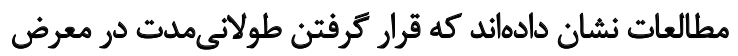

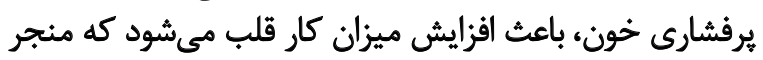

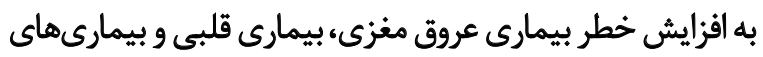

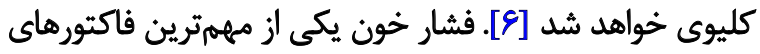

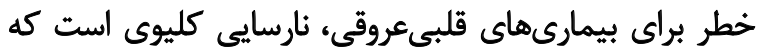

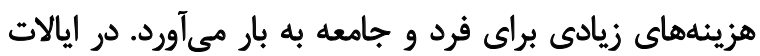

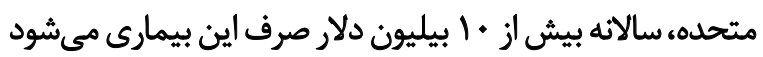

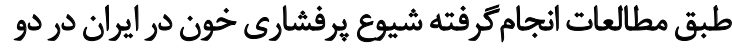

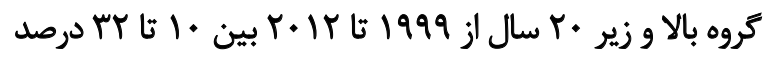

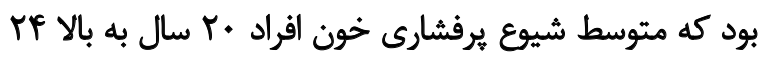

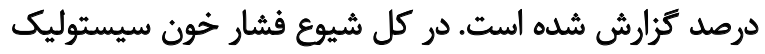

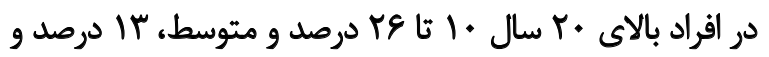

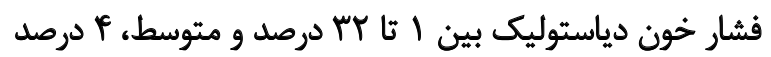

كزارش شده است [بآ]

\section{-}

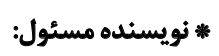

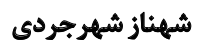
نشانى: اراك، داتشكاه اراك، دانشكده علوم ورزشى، كروه فيزيولوزيى و آسيب شناسى ورزشى.

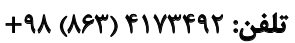
s-shahrjerdi@araku.ac.ir بست الكترونيكى 


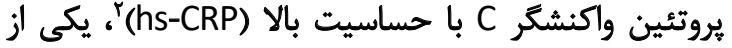

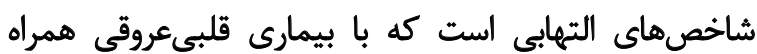

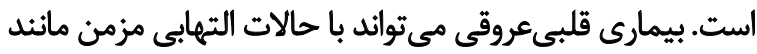

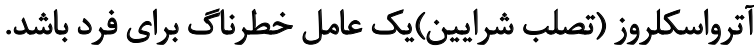

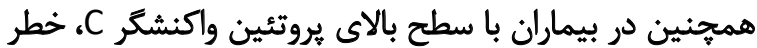

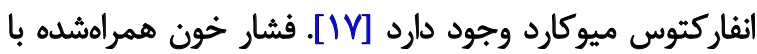

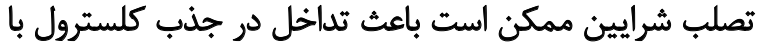

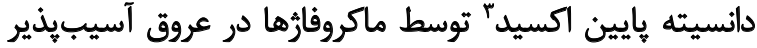

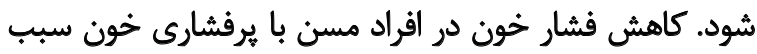

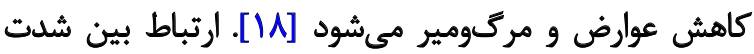

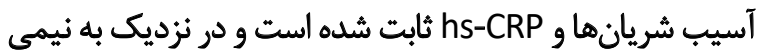

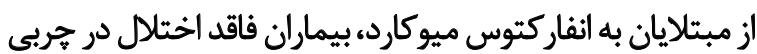

خون هستيند [19].

مطالعات مختلف و قابل دسترسى از تأثير ماسار بر فشار خون

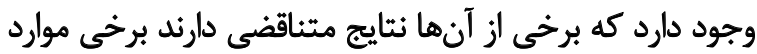

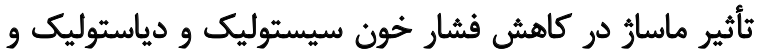

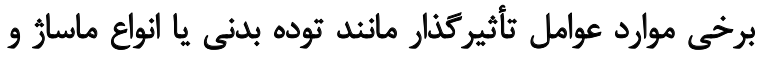

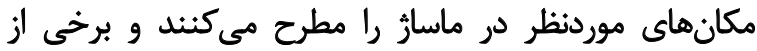

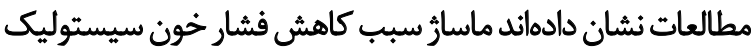

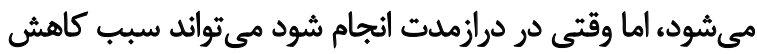

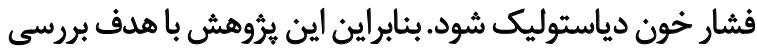

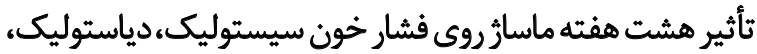

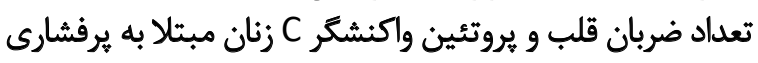

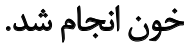

\section{مواد و روشها}

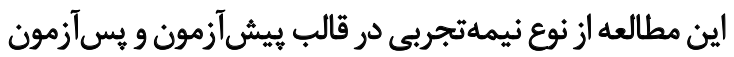

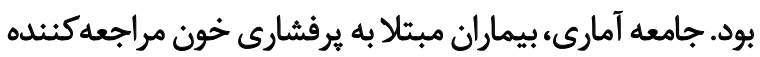

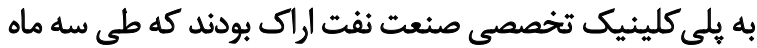

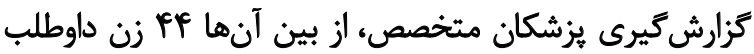

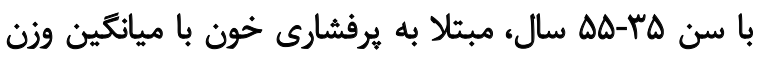

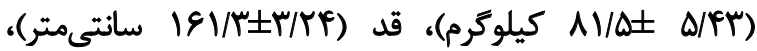

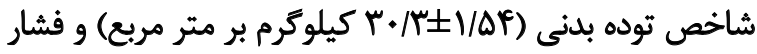

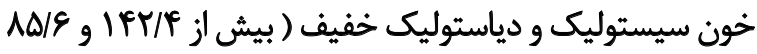

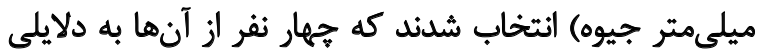

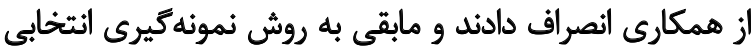

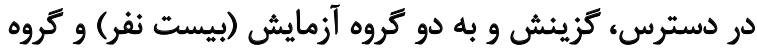

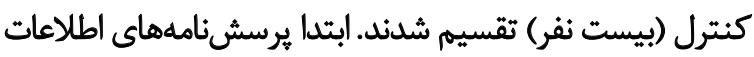

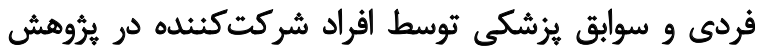

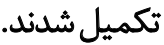

معيارهاى ورود به يُوهش: ابتلا به برفشارى خون بالا (فشار
[V] بر اساس مطالعات آمارى، يكينجم (N9) إدرصد) ايرانيان

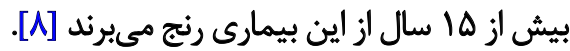
اولنى و همكاران مكمل درمانى را براى كاهش استرس و و

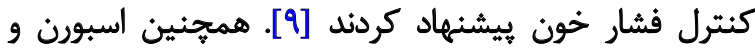

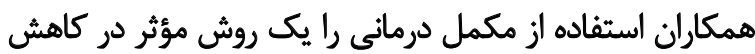

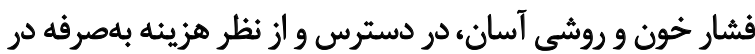

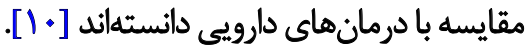

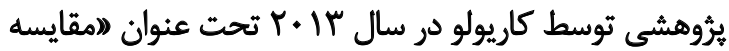

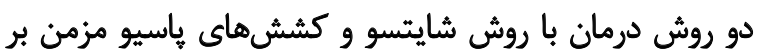

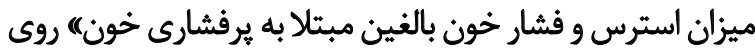

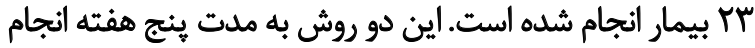

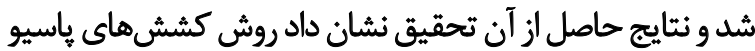

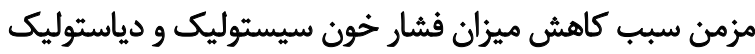

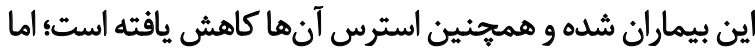
روش شايتسو تأثيرى بر آنها نداشته است [11 إنمان.

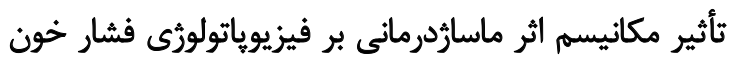

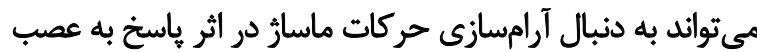

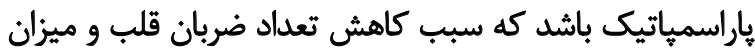

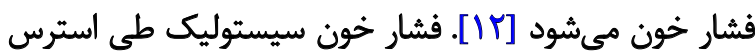

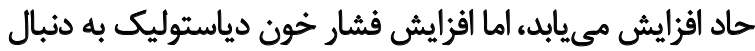

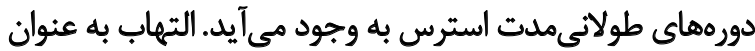

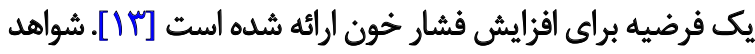

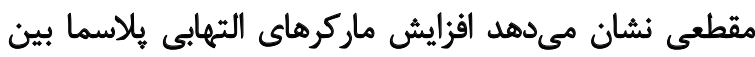

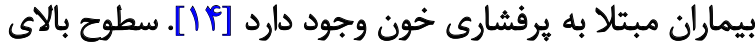

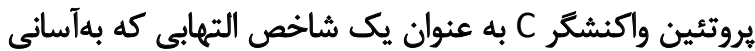

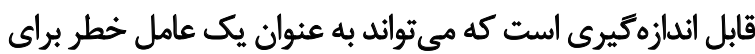

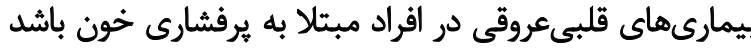

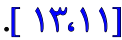

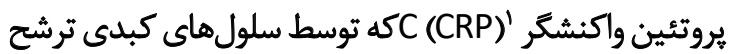

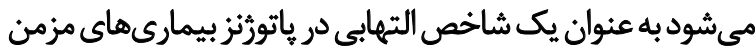

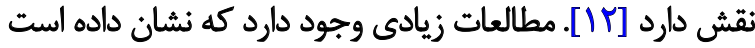

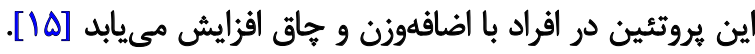

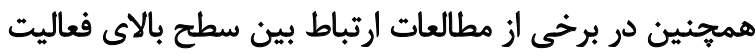

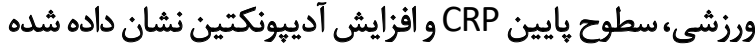

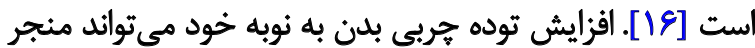

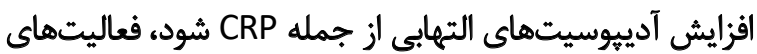

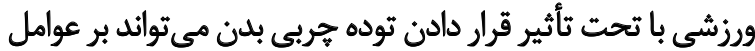

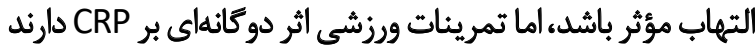

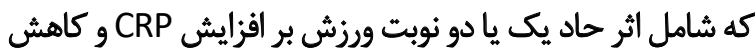

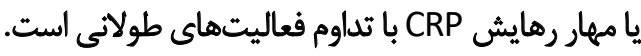




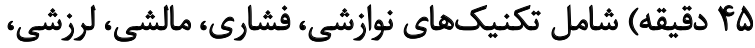

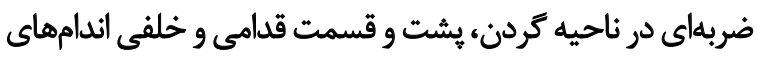
فوقانى و تحتانى بود كه به ترتيب ذيل ذيل انجام ترفت

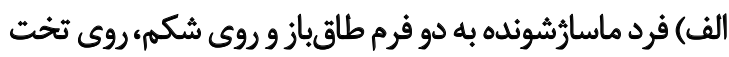
ماساز دراز كشيده و تكنيكها روى وم وم اعمال مى شيد.

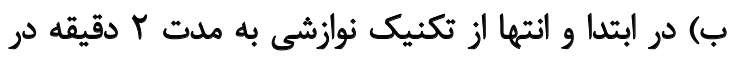
Fردن، f دقيقه در يشت، و A- A دقيقه در اندام فوقانى و تحتانى

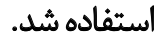

ج) تكنيك فشارى نيز در ناحيه تردن ب دقيقه، 8 دقيق دقيقه در

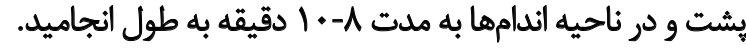

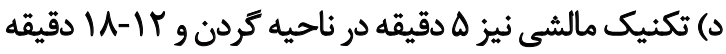

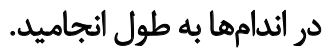

هـ) تكنيك لرزشى و ضربهاى نيز فقط در ناحيه يشت و اندامها به مدت 1-9 دقيقه انجام شد.

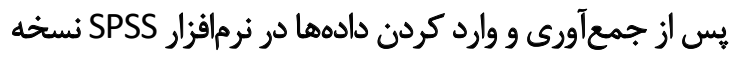

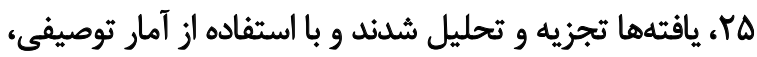

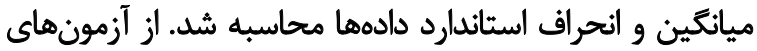

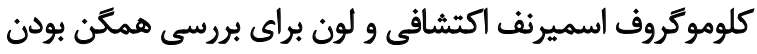

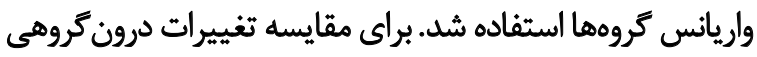

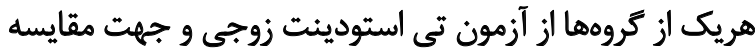

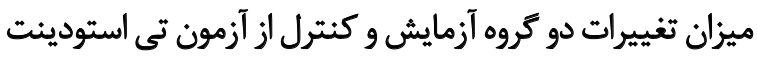

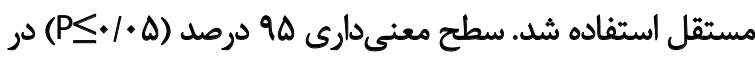

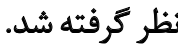

يافتهاهنا

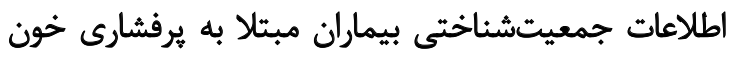

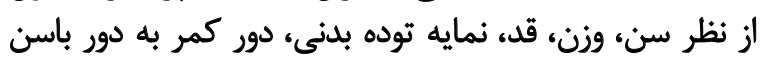

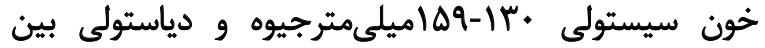

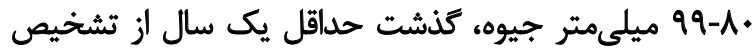

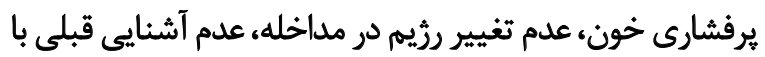

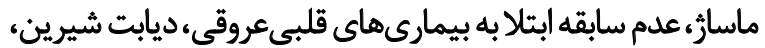

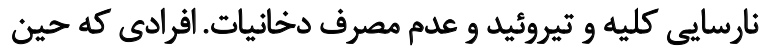

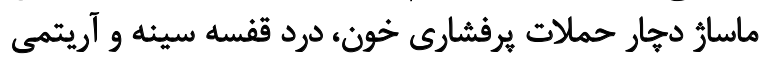

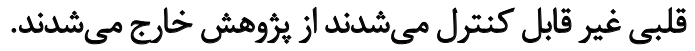
ابتدا به صورت شفاهى در يك جلسه به افراد شركت كنينده در

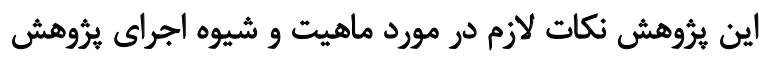

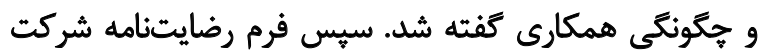

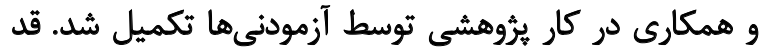

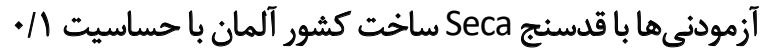

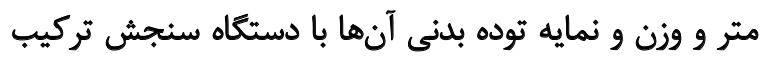

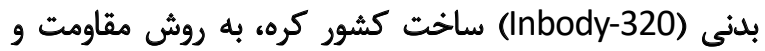

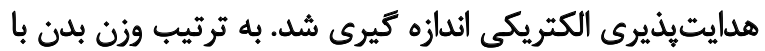

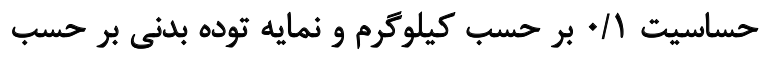

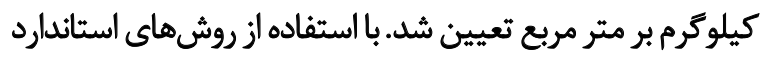

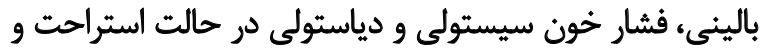
بدون استرس سه بار با فاصله ه دقيقه با فشارسنج

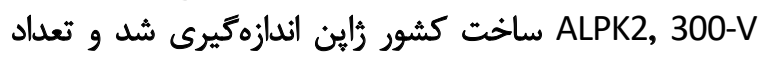

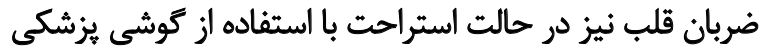

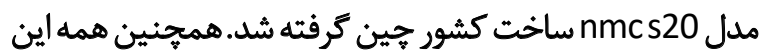

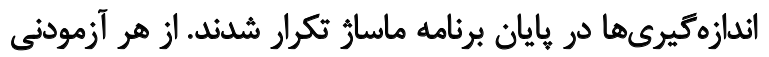

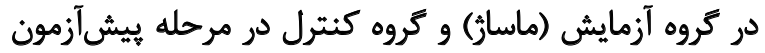

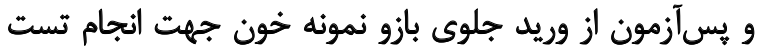

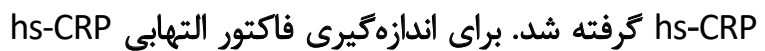

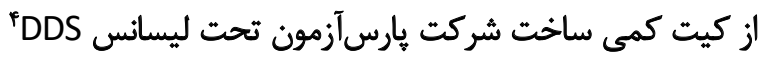
استفاده شد.

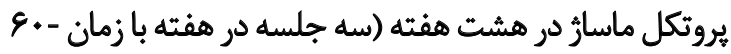

4. Diasys- Diagnostic-system, GMbH, Germany

جدول ا. اطلاعات جمعيتشناختى تروههاى موردمطالعه

\begin{tabular}{|c|c|c|}
\hline \multicolumn{2}{|c|}{ ميانكين+|نحراف معيار } & \multirow{2}{*}{ ويثوكىها } \\
\hline كروه كنترل (n=r) & كروه ماسار (+n=r) & \\
\hline$r \Delta / / \pm \Delta / / \varphi$ & $R T / M \pm \Delta / r)$ & سن(سال) \\
\hline$\Lambda \cdot(\Lambda \pm \Delta / r)$ & $V N \Delta \pm \Delta / V I$ & وزن (كيلوكرم) \\
\hline $1 \varepsilon+/ \Delta \pm r / \pi r$ & $\mid q f \pm r / \cdot r$ & قد (سانتى متر) \\
\hline$r / r \pm 1 / r$ & $r q / \Delta \pm \mid / \wedge 1$ & نمايه توده بلنى (كيلوكرم بر متر مربع) \\
\hline$q . / 1 r \pm q / \Delta r$ & $A V / T Y \pm g / T)$ & نسبت دور كمر بر دور باسن \\
\hline$f \cdot N \pm g(0)$ & $P H / \Delta \pm g / M$ & مدت زمان ابتلا به بيمارى (ماه) \\
\hline
\end{tabular}


جدول r. مقادير فشار خون، ضربان قلب كروه ماسار و كنترل در مراحل بيشآزمون و بيسآزمون

\begin{tabular}{|c|c|c|c|c|c|}
\hline \multirow{3}{*}{ تغييرات بين كروهى } & \multirow{3}{*}{ تغييرات درون كروهى } & & & \multirow{3}{*}{ كروه - اه } & \multirow{3}{*}{ مثغيرها } \\
\hline & & \multicolumn{2}{|c|}{ ميانكين \#انحراف معيار } & & \\
\hline & & يسأزمون & ييش أزمون & & \\
\hline \multirow{2}{*}{$\% \varphi^{\circ}$} & $\%$ & $\mid r \Delta / T \pm I T / F$ & $\mid \omega \cdot / \digamma \pm 10 / \%$ & 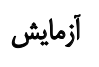 & \multirow{2}{*}{ 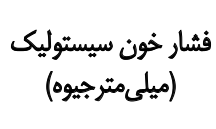 } \\
\hline &.$/ 9 \pi$ & $\| f \cdot / A \pm 1 \cdot / r$ & $\mid f \Delta / 8 \pm 1 . / \Delta$ & كنترل & \\
\hline \multirow{2}{*}{$\% 1^{\circ}$} &.. $.1^{\circ}$ & $q \cdot / r \pm \cdot / A r$ & $q \Delta / \Delta \pm \cdot / N$ & آزمايش & \multirow{2}{*}{ فشار خون دياستوليك } \\
\hline &.$/ M \varphi$ & $19 / r \pm . / 4$ & $q . / v \pm . / q \Delta$ & كتترل & \\
\hline \multirow{2}{*}{$.1 . .1^{\circ}$} & $1 ..)^{\circ}$ & $\Delta q \pm \varphi / m a$ & $1 \cdot \Delta \pm r / T \varphi$ & آزمايش & \multirow{2}{*}{ (تعداد در دقيقه) } \\
\hline & $\cdot / \Delta \Lambda$ & $q Y \pm r / Y q$ & $q \Delta \pm r / r q$ & كتنرل & \\
\hline
\end{tabular}

جدول r. مقادير بروتئين واكنشكر C كروه آزمايش و كثترل طى بيش آزمون و يس آزمون

\begin{tabular}{|c|c|c|c|c|c|}
\hline \multirow{2}{*}{ تغييرات بين تروهى } & \multirow{2}{*}{ تغييرات درونكروهى } & & & \multirow{3}{*}{ توره } & \multirow{3}{*}{ متغيرها } \\
\hline & & \multicolumn{2}{|c|}{ ميانكين \#انحراف معيار } & & \\
\hline سطح معنى دارى & سطح معنى دارى & يس آزمون & بيش آزمون & & \\
\hline \multirow{2}{*}{.. $.1^{*}$} & $1 . .1^{\circ}$ & $r / q) \pm 1 / F \varphi$ & $\Delta / \Delta \Delta \pm r / T f$ & تجربى & \multirow{2}{*}{$\begin{array}{l}\text { C (mg/L) يروتين واكنشخر } \\
\text { hs-CRP }\end{array}$} \\
\hline & . & $g(\Delta \pm r(\Delta)$ & $9 / \pm r / q$ & كتترل & \\
\hline
\end{tabular}

خون سيستوليك و دياستوليك زنان مبتلا به برفشارى خون تأثير

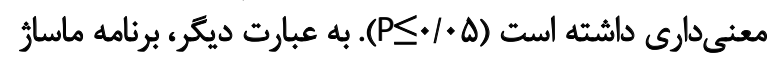

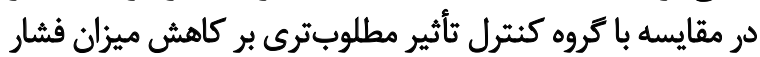

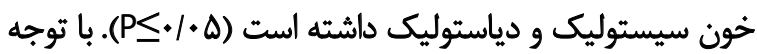

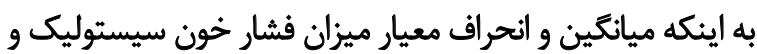

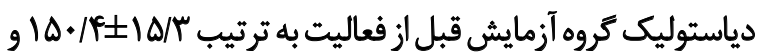

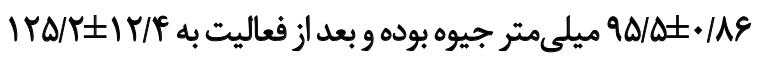

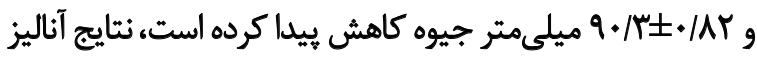

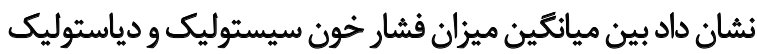

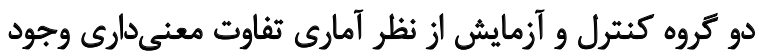

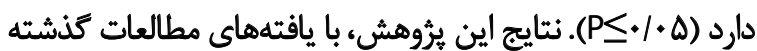

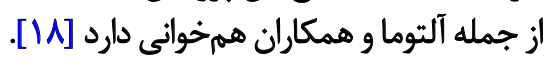

بح

در اين يُروهش فشار خون سيستوليك و دياستوليك در كروه

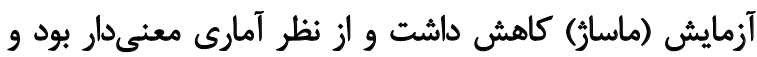

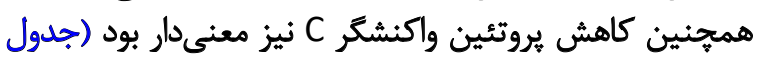

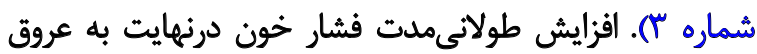

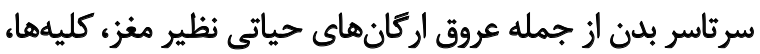

ه و مدتزمان ابتلا به بيمارى در جدول شماره آلورده

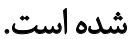
يافتهها در دو كروه نشان داد كه هيج كونه اختلاف معنى دارى

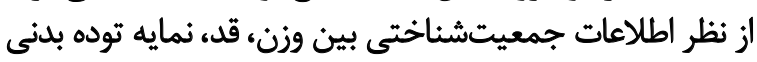

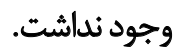

در جدول شماره Y ميزان فشار خون سيستوليك و دياستوليك

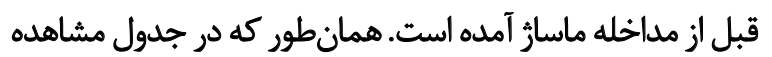

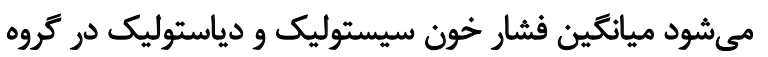

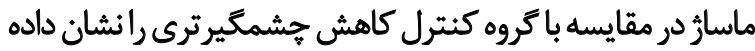

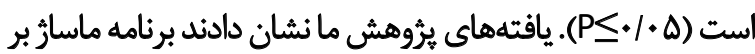

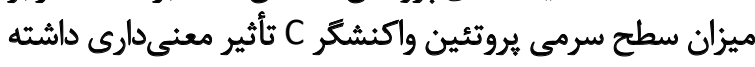

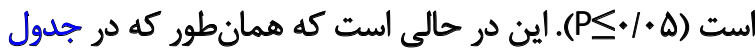

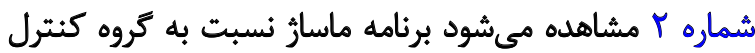

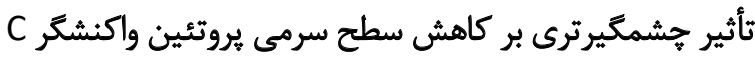

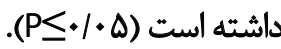
جدول شماره Y نشان مي دهد كه برنامه ماساز بر ميزان فشار 5. Waist-hip Ratio 
همسو بود. آنها اثر ماسار عميق را بر ميزان فشار خون و و تعداد

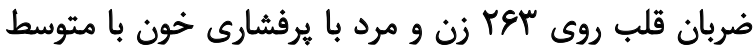

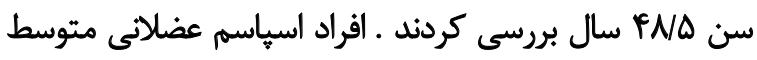

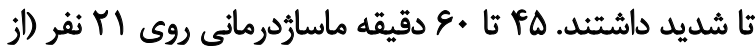

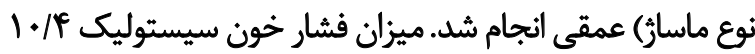

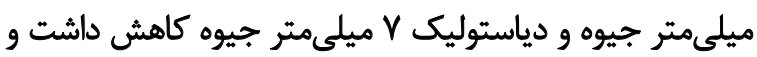

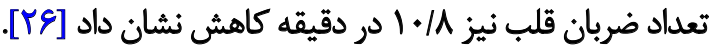

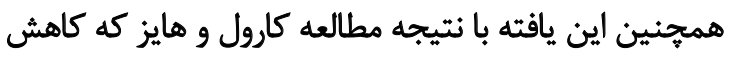

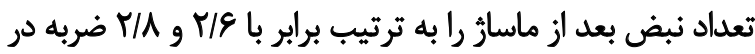

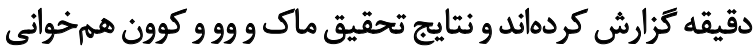

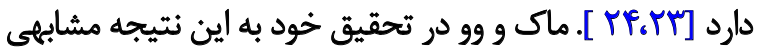

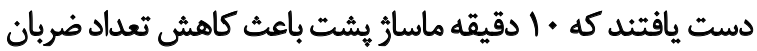

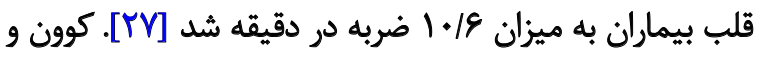
همكاران هم كاهش نبض را به مقدار ه/ ه دقيقه كزارش كردند

[r人]

ثأثير هشت هفته ماسار بر ميزان بروتئين واكنشكر Cبا بروهش

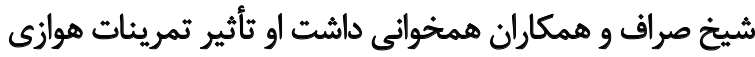

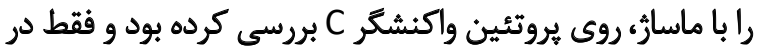

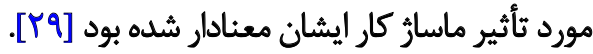

در اين يزوهش با انجام هشت هفته ماساز به واسطه كاهش

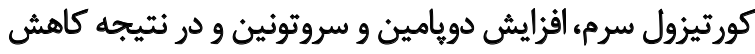

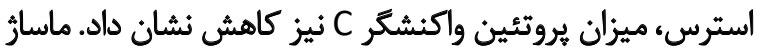

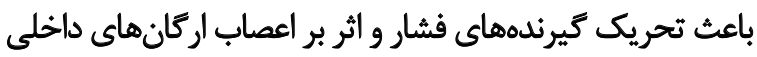

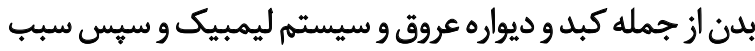

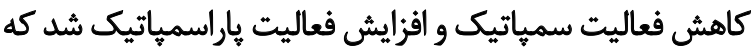

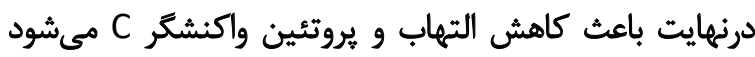

[r·]

\section{نتيجهيرى}

درمانهاي مختلفى براي كاهش فشار خون در افراد مبتلا به

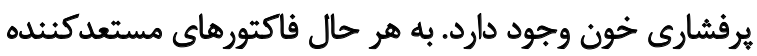

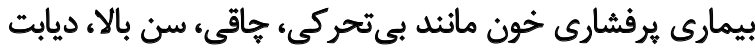

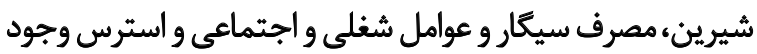

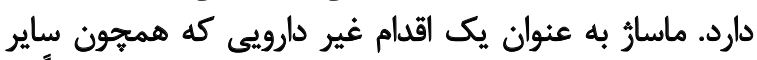

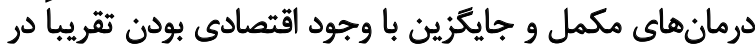

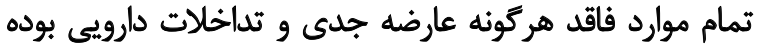

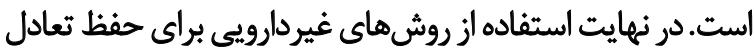

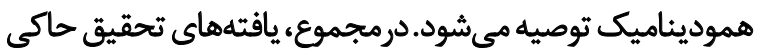

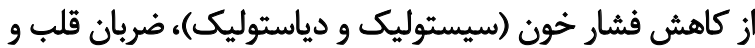
يروتئين واكنشكر C در كروه ماسار بوده است.

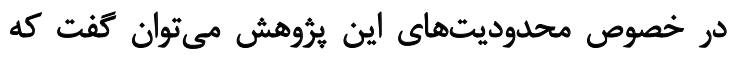

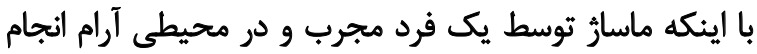

قلب و جشمها آسيب وارد مي كند. بنابراين اثرهاى معمول

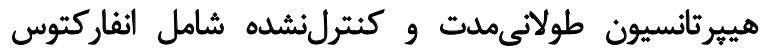

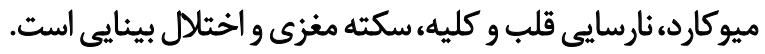

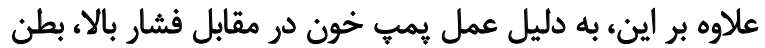

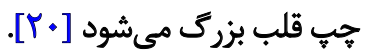

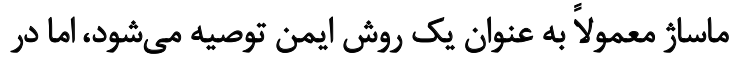

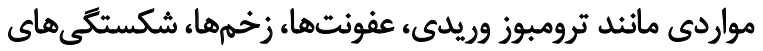

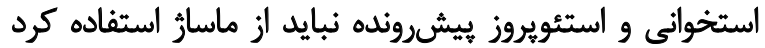

با توجه به اينكه ميانكين و انحراف معيار ميزان فشار خون

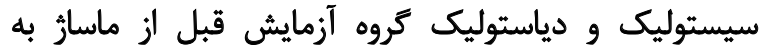

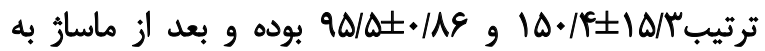

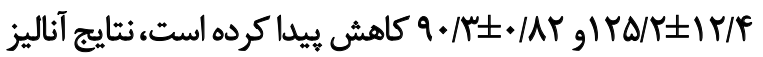

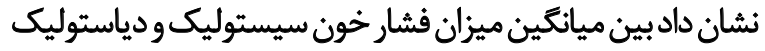

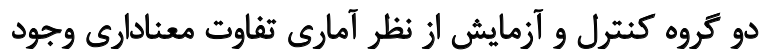

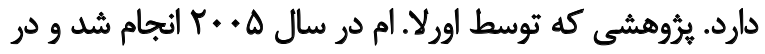

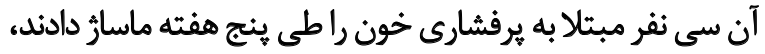

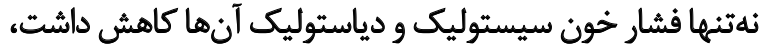

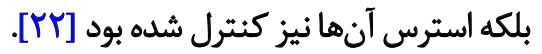

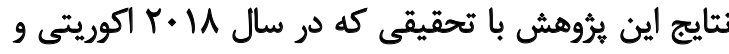

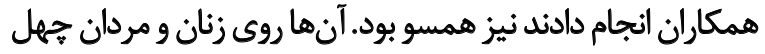

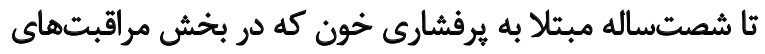

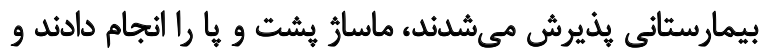

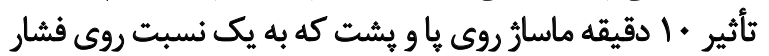

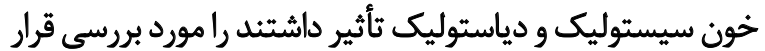

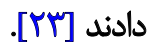

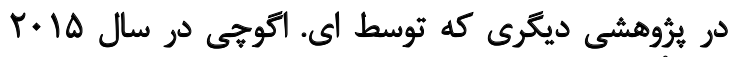

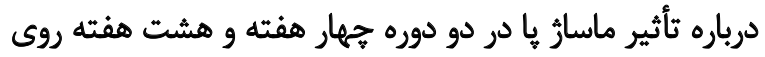

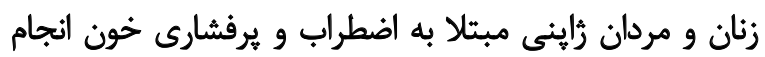

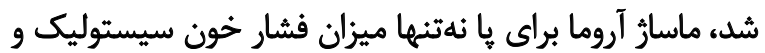

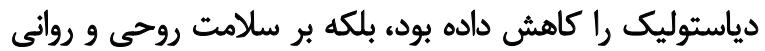

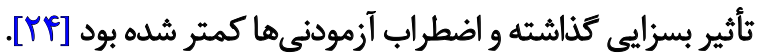

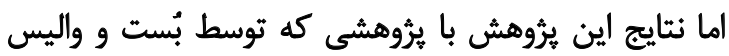

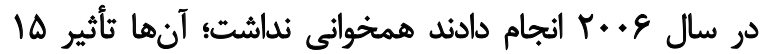

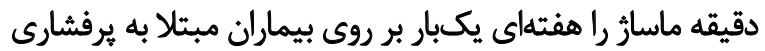

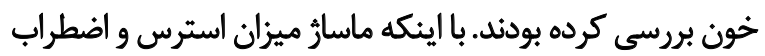

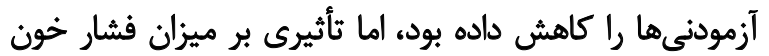

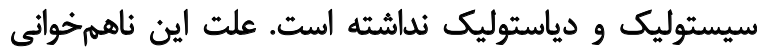

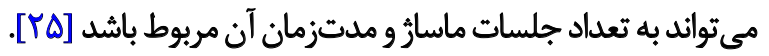

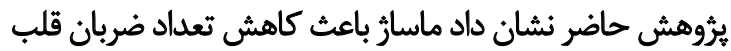

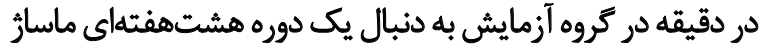

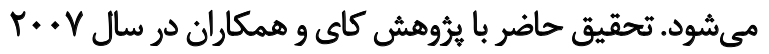




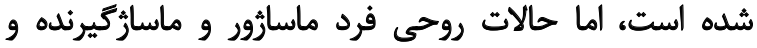

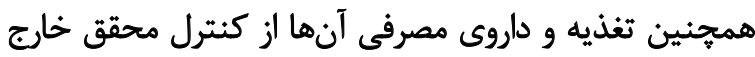

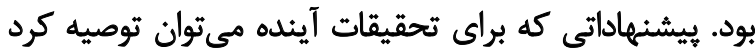

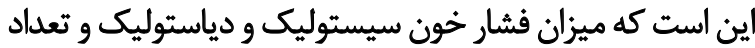

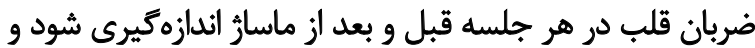

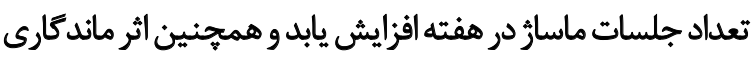
ماسار مورد بررسى قرار كيرد.

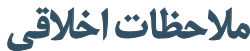

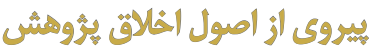

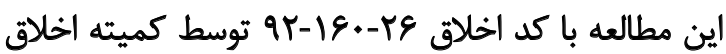
دانشَاه علوميزشكى اراك به تصويب رسيده است.

$$
\text { مامي مالى }
$$

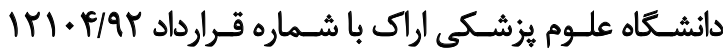

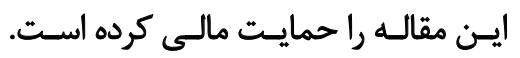

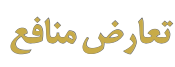

نويسندكان مقاله هيجكونه تعارضى در منافع اعلام نكردند.

$$
\text { تشائرو وتشكر }
$$

از تلاشهاي همكارانم در يلى كلينيك صنعت نفت استان

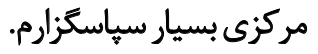




\section{References}

[1] NCD Risk Factor Collaboration. Worldwide trends in blood pressure from 1975 to 2015: A pooled analysis of 1479 population-based measurement studies with 19.1 million participants. Lancet. 2017; 389(10064):37-55. [DOI:10.1016/S0140-6736(16)31919-5]

[2] Chow CK, Teo KK, Rangarajan S, Islam S, Gupta R, Avezum A, et al. Prevalence, awareness, treatment, and control of hypertension in rural and urban communities in high-, middle-, and low-income countries. J Am Med Assoc. 2013; 310(9):959-68. [DOI:10.1001/ jama.2013.184182] [PMID]

[3] Mohsenzadeh Y, Motedayen M, Hemmati F, Sayehmiri K, Sarokhani MT, Sarokhani D. Investigating the prevalence rate of hypertension in Iranian men and women: A study of systematic review and metaanalysis. J Basic Res Med Sci. 2017; 4(1):53-62. [DOI:10.18869/acadpub.jbrms.4.1.53]

[4] Rahimian Mashhad Z, Attarzade Hoseyni SR, Aryan Nejhad J. [The effect of aerobic training and diet on cardiovascular risk factors and blood pressure in overweight and obese women with hypertension (Persian)]. Iran J Endocrinol Metab. 2010; 12(4):376-384. http://ijem. sbmu.ac.ir/article-1-972-fa.html

[5] Khoshdel AR, Dormanesh B, Noorifard M. [Ups and downs of hypertension (Persian)]. Ann Mil Health Sci Res. 2011; 9(2):118-29. https:// www.sid.ir/fa/journal/ViewPaper.aspx?id=137047

[6] Bush JG. Post-exercise hypotension in brief exercise [MSc. thesis]. Bowling Green, KY: Western Kentucky University; 2011. https://digitalcommons.wku.edu/theses/1072/

[7] Goodarzi MR, Ghanbari MR, Badakhsh M, Masinaeinezhad N, Abbaszadeh M. A study on hypertension in Zabol population over 18 years old (Persian)]. Iran J Endocrinol Metab. 2003; 4(4):183-90. http://ijem.sbmu.ac.ir/article-1-578-fa.pdf

[8] Khosravi AR, Mohammadi FN, Shahrokhi S, Shahin Sh, Rezvan A. [Drugs to patients with hypertension in central areas of Iran (Persian)]. J Isfahan Med Sch. 2004; 22(74-75):100-8. https://www.sid.ir/ fa/journal/ViewPaper.aspx?id=31641

[9] Bermudez EA, Rifai N, Buring J, Manson JE, Ridker PM. Interrelationships among circulating IL-6, C-reactive protein, and traditional cardiovascular risk factors in women. Arterioscler Thromb Vasc Biol. 2002; 22:1668-73. [DOI:10.1161/01.ATV.0000029781.31325.66] [PMID]

[10] Sesso HD, Buring JE, Rifai N, Blake GJ, Gaziano JM, Ridker PM. C-reactive protein and the risk of developing hypertension. J Am Med Assoc. 2003; 290(22):2945-51. [DOI:10.1001/jama.290.22.2945] [PMID]

[11] Carielo AA, Nunes RAM, Pernambuco CS, Diré GF, Silva JG, Amorin FDS, et al. Comparison of the chronics effecs of a passive stretching program and shiatsu-therapy on the stress phase and blood pressure levels of hypertension adults. Am J Res Commun. 2013; 1(12):24253. http://www.usa-journals.com/wp-content/uploads/2013/11/Carielo_Vol112.pdf

[12] Niskanen L, Laaksonen DE, Nyyssonen K, Punnonen K, Valkonen VP, Fuentes R, et al. Inflammation, abdominal obesity, and smoking as predictors of hypertension. Hypertens. 2004; 44(6):859-65. [DOI:10.1161/01.HYP.0000146691.51307.84] [PMID]

[13] Choi J, Joseph L, Pilote L. Obesity and C-reactive protein in various populations: A systematic review and meta-analysis. Obes Rev. 2012; 14(3):232-44. [DOI:10.1111/obr.12003] [PMID]

[14] Taube A, Schlich R, Sell H, Eckardt K, Eckel J. Inflammation and metabolic dysfunction: Links to cardiovascular diseases. Am J Physiol-
Heart Circ. 2012; 302(11):148-65. [DOI:10.1152/ajpheart.00907.2011] [PMID]

[15] Hamer M, Steptoe A. Walking, vigorous physical activity, and markers of hemostasis and inflammation in healthy men and women. Scand J Med Sci Sports. 2008; 18(6):736-41. [DOI:10.1111/j.16000838.2007.00747.x] [PMID]

[16] Abramson JL, Vaccarino V. Relationship between physical activity and inflammation among apparently healthy middle-aged and older US adults. Arch Intern Med. 2002; 162(11):1286-92. [DOI:10.1001/ archinte.162.11.1286] [PMID]

[17] Adukauskiene D, Ciginskiene A, Adukauskaite A, Pentiokiniene D, Šlapikas R, Ceponiene I. Clinical relevance of high sensitivity Creactive protein in cardiology. Medicina (Kaunas). 2016; 52(1):1-10. [DOI:10.1016/j.medici.2015.12.001] [PMID]

[18] Al-Tu'maa FJ, Abd-Yasera ZA, Al-Naffi KO. Association between hsCRP levels and the severity of coronary atherosclerosis. J Contemp Med Sci. 2016; 2(6):42-44. http://www.jocms.org/index.php/jcms/ article/view/70

[19] Furuhashi M, Saitoh S, Shimamoto K, Miura T. Fatty Acid-Binding Protein 4 (FABP4): Pathophysiological insights and potent clinical biomarker of metabolic and cardiovascular diseases. Clin Med Insights Cardiol. 2015; 8(Suppl III):23-33. [DOI:10.4137/CMC.S17067] [PMID] [PMCID]

[20] Brunner LS, Smeltzer SC, Bare BG, Hinkle JL, Cheever KH. Brunner and Suddarth's textbook of medical-surgical nursing [AH. Pishgoee, Persian trans]. Tehran: Boshra; 2008.

[21] Ernst E, Pittler MH, Stevinson C, White A. The desktop guide to complementary and alternative medicine: An evidence-based approach. London: Mosby International Ltd; 2001.

[22] Aourell M, Skoog M, Carleson J. Effects of Swedish massage on blood pressure. Complement Ther Clin Pract. 2005; 11(4):242-6. [DOI:10.1016/j.ctcp.2005.02.008] [PMID]

[23] Biswas S, Asokan R, Lenka A, Patro S. A comparative study to assess the effectiveness of foot massage $\&$ back massage in reducing blood pressure among hypertensive patients admitted in medicine ward attertiarycare hospital, Bhubaneswar. IOSR J Nurs Health Sci. 2019; 7(1 ):1-6. [DOI:10.9790/1959-0701010106]

[24] Eguchi E, Funakubo N, Tomooka K, Ohira T, Ogino K, Tanigawa $T$. The effects of aroma foot massage on blood pressure and anxiety in Japanese community-dwelling men and women: A crossover randomized controlled trial. PLoS One. 2016; 11(3):e0151712. [DOI:10.1371/journal.pone.0151712] [PMID] [PMCID]

[25] Bost N, Wallis M. The effectiveness of a 15 minute weekly massage in reducing physical and psychological stress in nurses. Aust J Adv Nurs. 2006; 23(4):28-33. https://www.researchgate.net/publication/6984749

[26] Kaye AD, Kaye AJ, Swinford J, Baluch A, Bawcom BA, Lambert TJ, et al. The effect of deep-tissue massage therapy on blood pressure and heart rate. J Altern Complement Med. 2008; 14(2):125-8. [DOI:10.1089/acm.2007.0665] [PMID]

[27] Mok E, Pang Woo C. The effects of slow-stroke back massage on anxiety and shoulder pain in elderly stroke patients. Comple ment Therap Nurs Midwifery. 2004; 10(4):209-16. [DOI:10.1016/j. ctnm.2004.05.006] [PMID]

[28] Cowen VS, Burkett L, Bredimus J, Evans DR, Lamey S, Neuhauser T, et al. A comparative study of Thai massage and Swedish massage relative to physiological and psychological measures. J Bodyw Mov Ther. 2006; 10(4):266-75. [DOI:10.1016/j.jbmt.2005.08.006] 
[29] Sheikhsaraf B, Peeri M, Azarbayjani M, Agha-Alinejad H. The effect of aerobic interval training and massage therapy on $\mathrm{C}$-reactive protein and cardiorespiratory fitness in cardiovascular patients after coronary artery bypass graft. Ann Appl Sport Sci. 2016; 4(1):9-16.

[DOI:10.7508/aass.2016.01.003]

[30] Billhult A, Lindholm C, Gunnarsson R, Stener Victorin E. The effect of massage on immune function and stress in women with breast cancer-a randomized controlled trial. Auton Neurosci. 2009; 150(12):111-5. [DOI:10.1016/j.autneu.2009.03.010] [PMID] 
This Page Intentionally Left Blank 